\title{
Erratum
}

7. Gröber U, Spitz J, Reichrath J, Kisters K, Holick MF (2013) Vitamin D: Update 2013: from rickets prophylaxis to general preventive healthcare. Dermatoendocrinol 5(3):331-347

8. Herold G (2012) Innere Medizin. Eigendruck, Köln, S 719

9. Holick MF (2007) Vitamin D deficiency. N Engl J Med 357(3):266-281

10. Jakob F (2007) Metabolische Knochenerkrankungen. Internist 48:1101-1117

11. Jakob F (2005) Primary and secondary osteoporosis. The important role of internal medicine in its differential diagnosis. Internist 46:524-530

12. Maier GS, Jakob $P$, Horas $K$, Roth $K E$, Kurth $A A$, Maus U (2013) Vitamin D deficiency in orthopaedic patients: a single center analysis. Acta Orthop Belg 79(5):587-591

13. Pfeilschifter J (2006) 2006 DVO-guideline for prevention, diagnosis, and therapy of osteoporosis for women after menopause, for men after age 60 executive summary guidelines. Exp Clin Endocrinol Diagetes 114:611-622

14. Reuss-Borst (2014) Metabolische Knochenkrankheit Osteomalazie. Z Rheumatol 73:316-322

15. Richter WO, Eckartstein von A (2005) Vitaminstoffwechsel. In: Siegenthaler W, Huber E (Hrsg) Klinische Pathophysiologie. Thieme, Stuttgart, S 234

16. Saghafi M, Azarian A, Hashemzadeh K, Sahebari $M$, Rezaieyazdi Z (2013) Bone densitometry in patients with osteomalacia: is it valuable? Clin Cases Miner Bone Metab 10(3):180-182

17. Zichner L, Enderle A (2003) Osteomalazie. In:Wirth CJ, Zichner L (Hrsg) Orthopädie und orthopädische Chirurgie - Systemerkrankungen. Thieme, Stuttgart, S 163-165
Orthopäde $2015 \cdot 44: 702$

DOI 10.1007/s00132-015-3151-7

Online publiziert: 22. August 2015

(c) Springer-Verlag Berlin Heidelberg 2015

CrossMark

M. Salzmann ${ }^{1} \cdot$ C. Krohn ${ }^{2} \cdot$ N. Berger ${ }^{1}$

${ }^{1}$ Kinderorthopädie, Klinikum Schwabing, TUM Rechts der Isar, Städtisches Klinikum München GmbH, München, Deutschland

${ }^{2}$ Kinderchirurgie Klinikum Schwabing, München, Deutschland

\section{Erratum zu: Osteogenesis imperfecta}

Erratum zu:

Orthopäde (2014)

43:764-771

DOI:10.1007/s00132-013-2229-3

In der vorliegenden Übersicht wurde nicht bzw. nicht ausreichend kenntlich gemacht, dass die Arbeit sowohl strukturell als auch inhaltlich in Teilen basiert auf

T. Wirth (2012) Osteogenesis imperfecta Orthopäde 41:773-784

und

L. Zeitlin, F. Fassier, FH Glorieux (2003) Modern approach to children with osteogenesis imperfecta. J Pediatr Orthop B 12:77-87

Die korrespondierende Autorin bittet sowohl die Leserinnen und Leser als auch die Autoren der beiden Arbeiten, dies zu entschuldigen.

\section{Korrespondenzadresse}

\section{Dr. M. Salzmann}

Kinderorthopädie, Klinikum Schwabing

TUM Rechts der Isar, Städtisches Klinikum

München $\mathrm{GmbH}$

Kölner Platz 1, 80804 München

maya.salzmann1@klinikum-muenchen.de
Die Onlineversion des Originalbeitrages ist erreichbar unter doi:10.1007/s00132-013-2229-3 